\title{
Effect of Binaural Stimulation on Attention and EEG
}

\author{
Adela CRESPO $^{(1)}$, Manuel RECUERO ${ }^{(1)}$, Gerardo GALVEZ $^{(1),(2)}$, Adrián BEGOÑA ${ }^{(1)}$ \\ (1) Centro de Acústica Aplicada y Evaluación no Destructiva, \\ Consejo Superior de Investigaciones Cientificas (CAEND-CSIC) \\ C/ Serrano 144, 28005 - Madrid, Spain; \\ e-mail: adela.crespo@csic.es; manuel.recuero@upm.es; gerardo.galvez@csic.es; adrian.begona@csic.es \\ (2) Centro de Tecnología Biomédica, \\ Universidad Politécnica de Madrid (CTB-UPM) \\ Campus de Montegancedo, 28223 - Pozuelo de Alarcón, Spain; \\ e-mail: gerardo.galvez@ctb.upm.es \\ (received December 4, 2012; accepted November 2, 2013)
}

\begin{abstract}
When two pure tones of slightly different frequency are presented separately to each ear, the listener perceives a third single tone with amplitude variations at a frequency that equals the difference between the two tones; this perceptual illusion is known as the binaural auditory beat (BB). There are anecdotal reports that suggest that the binaural beat can entrain EEG activity and may affect the arousal levels, although few studies have been published.

There is a need for double-blind, well-designed studies in order to establish a solid foundation for these sounds, as most of the documented benefits come from self-reported cases that could be affected by placebo effect. As BBs are a cheap technology (it even exists a free open source programmable binauralbeat generator on the Internet named Gnaural), any achievement in this area could be of public interest. The aim in our research was to explore the potential of BBs in a particular field: tasks that require focus and concentration. In order to detect changes in the brain waves that could relate to any particular improvement, EEG recordings of a small sample of individuals were also obtained.

In this study we compare the effect of different binaural stimulation in 7 EEG frequency ranges. 78 participants were exposed to 20-min binaural beat stimulation. The effects were obtained both qualitative with cognitive test and quantitative with EEG analysis. Results suggest no significant statistical improvement in 20-min stimulation.
\end{abstract}

Keywords: binaural auditory beats, attention, frequency following response.

PACS no.: 43.66.Pn

\section{Introduction}

Binaural waves stimulation at different frequencies has been used in the past two decades in the treatment of many diseases and also to modify different states such as pain, relaxation, meditation, anxiety, and also to improve attention and memory.

Binaural tones are subjective auditory sensations which occur as a result of receiving two tones of slightly different frequencies, each in a different ear. Binaural waves were discovered by Heinrich Wilhelm Dove in 1839.

The binaural hearing beats occur in the brain stem in response to auditory stimulation produced by two pure tones of slightly similar frequency, each in a different ear. The upper grove located in the brainstem is responsible for interpreting the frequency difference, which is called the binaural tone (Oster, 1973). For example, if we issue a $110 \mathrm{~Hz}$ tone in the right ear and another $115 \mathrm{~Hz}$ tone in the left ear, the frequency difference between the two will be $5 \mathrm{~Hz}$, and this is the binaural tone (LANE et al., 1998).

The ability of a human to hear binaural beats seems to be a result of evolutionary assimilation. Many advanced species can detect binaural beats, depending on a skull size (KASPRZAK, 2011).

The binaural tones are the result of neuronal firing overlapping at an appropriate level of the auditory 
path, coming from the right and left ear. The binaural tones show how neuronal firing in the auditory nerve maintains the phase information of the received signal (KASPRZAK, 2011; OzIMEK et al., 2008). The route of the auditory nerve to the brain allows the exchange of information coming from both sides, before the sound reaches the cortex, ensuring conscious listening. This exchange occurs at least in two areas of the auditory nerve: in the upper olive grove body, small mass of gray matter located in the ventral pontine reticular system (in charge of contralateral integration of auditory system), and transfers it to other area, the inferior colliculus (Schwarz, TAYLOR, 2005). The two signals arriving from different ears are connected in the brain, resulting in a third signal called the binaural tone (KASPRZaK, 2011; PAdmanabhan et al., 2005).

Listening to binaural beats provides the information to the network system, also called the diffuse activation system, a large area of the brain that looks like a network, which makes decisions about the clarity, concentration, and awareness. If either internal (feelings, behaviors, or beliefs) or external (perceived by the senses) stimuli are not in conflict with information willing, the reticular system modifies the activity of brain waves, adjusting these to the frequency binaural tone. This is a natural function of homeostasis. The brain regulates automatic body functions to maintain homeostasis. The reticular system tries to maintain homeostasis in a natural way, controlling and maintaining sustainable states of brain wave activity all the time (unless you get external or internal information). Thanks to the fact that the frequency characteristics of the auditory signal and the frequency of brain waves are similar, the reticular system begins processing the information coming from the auditory signal, believing that the information coming in binaural tone comes of brain wave activity (Sмiтh et al., 1975; WAHBeH et al., 2007). The term used for this synchronization process in the literature is "entrainment".

With the development of EEG, it became increasingly clear that certain frequencies can induce changes in the EEG, for instance binaural waves in the delta range $(1-4 \mathrm{~Hz})$ are associated with sleep, those in the theta range $(4-8 \mathrm{~Hz})$ relate to a slow brain activity, while those that are in the alpha range are associated with awake states $(8-13 \mathrm{~Hz})$. The binaural beats in the beta range (16-24 Hz) occur in states of alertness and concentration (LANE et al., 1998).

Foster (1991) examined the effects of stimulation in the alpha range (FosTer, 1990), combined with neurofeedback in this range. The results of this study suggest that the combination of binaural tones with neurofeedback result in increased production of alpha comparing with application of neurofeedback only, but also the group receiving only binaural stimulation waves had higher alpha production than any of the groups. LANE et al. (1998) provide evidence on the fre- quency response of runoff to $7 \mathrm{~Hz}$ and its direct effect on psychomotor development and mood.

C. Kasprzak examined experimentally the effect of a binaural wave in 20 subjects (KASPRZAK, 2011). The carrier frequency was $100 \mathrm{~Hz}, 73 \mathrm{~dB}$ SPL, with 20 minutes of binaural stimulation. In this study, positive statistical results are obtained on the modification of cortical arousal with binaural frequencies. Also an entrainment at $10 \mathrm{~Hz}$ for 4 of the subjects was found.

Among many applications that are commercial, binaural waves seem to help achieve deeper and faster meditative states. Meditation techniques are used to achieve altered states of consciousness, developing awareness and perception, reduce stress, and increase a positive attitude (BARUŠs, 2001). We found specific patterns in the EEG of meditators when compared with subjects who have never meditated as well when compared against baseline state (AFTANAS, Golocheikine, 2002).

LAVALleE and Koren (2011) conducted a study with 8 subjects, of whom half were expert meditators and half rookies, subjected to two different binaural stimulation, $7 \mathrm{~Hz}$ to facilitate meditation and the other $15 \mathrm{~Hz}$ to hinder meditation (LAVAlleE et al., 2011). The results evidenced that novices had less power theta and increased gamma in $15 \mathrm{~Hz}$ condition. This result suggest experienced meditators have developed techniques, over the years of practice, to maintain a deep state of meditation while blocking external stimuli (LAVALLEE et al., 2011).

Susan KenNel (2010) studied the effectiveness of binaural stimulation to reduce symptoms of inattention in teenagers. The study confirmed the utility of binaural waves. It was a randomized, double blind and placebo control study with 20 young people who listened to 20 min stimulation, 3 times per weeks during 3 weeks. They used TOVA, Color Trails test and Homework Problem Checklist to evaluate the cognitive change. They didn't find any significant attention results despite the feedback from parents was very positive (KENNEL et al., 2010).

There were similar studies that found positive results on attention tasks (LANE et al., 1998).

There is a need for double-blind, well-designed studies in order to establish a solid foundation for these sounds, as most of the documented benefits come from self-reported cases that could be affected by placebo effect. As BBs are a cheap technology (it even exists a free open source programmable binaural-beat generator on the internet named Gnaural), any achievement in this area could be of public interest. The aim in our research was to explore the potential of BBs in a particular field: tasks that require focus and concentration. In order to detect changes in the brain waves that could relate to any particular improvement, EEG recordings of a small sample of individuals were also obtained. 


\section{Materials and methods}

\subsection{Study design}

This study is a randomized, double blind, placebo controlled exploratory pilot investigation in order to determine the effect of two different binaural beat stimulations in the theta and beta ranges and therefore establish the start methodology to continue research in this area of interest.

We have divided experiments into two parts, $\mathrm{A}$ and B; in Experiment $\boldsymbol{A}$ we measured the improvement in attention with different tests, while in Experiment $\boldsymbol{B}$ we measured EEG modifications.

\subsection{Setting a sample}

Experiment A: We have recruited 60 members of general public from Madrid, included men and women (28 females and 32 males; mean $( \pm$ s.d.) aged $28.9 \pm 4.3$ years) who were new to binaural beat stimulation and who consented to participate. Exclusion criteria were neurological diseases and left-handedness. They were informed about the general goal of the research and rules of their particular experiment, and completed an audiometric test to assure they suffered no major hearing loss.

Experiment B : We have recruited 18 members of general public from Madrid (5 females and 13 males, aged $26.6 \pm 7.49$ years), who were new to binaural beat stimulation and who consented to participate. Exclusion criteria were neurological diseases and righthandedness. They were informed about the general goal of the research and rules of their particular experiment, and completed an audiometric test to assure they suffered no major hearing loss.

\subsection{Procedures}

Experiment A: Participants were blindly allocated to one of three groups according to a predetermined computer-generated random sequence. They listened for 20 minutes via standard headphones to a commercial binaural audio beat (Binaural commercial Group, $n=20)$, an identical soundtrack without these tones (Placebo Group, $n=20$ ), or a self-made audio including several layers of BBs (Binaural experimental Group, $n=20$ ). The commercial beat audio had the BBs embedded in a fluctuating pink noise that was used also in the self-made audio (the noise that all the Placebo Group listened to). All were instructed to relax and listen with closed eyes in a comfortable position. After 20 minutes, the participants were asked to open their eyes and, without removing the headphones, were requested to complete three different tasks: the test on differences perception (REPÁRAZ et al., 1996), used to evaluate attention and perceptive skills; the 5 digit test, used to measure the processing speed of the subjects and their ability to direct and switch their attention control; and the EMAV-2, used to measure sustained attention and quality of attention.

When the three tests were completed, headphones were removed and participants were questioned about any unusual feeling during the course of the experiment.

Experiment B: Participants were blindly allocated to one of three groups according to a predetermined computer-generated random sequence. They listened for 20 minutes via standard headphones to a commercial binaural beat audio (Binaural commercial Group, $n=6$ ), an identical soundtrack without these tones (Placebo Group, $n=6$ ), or a self-made audio including several layers of BBs (Binaural experimental Group, $n=6$ ). EEG was used in this study, as we were interested in changes evoked by BBs, and data from 3-min period prior to listening were firstly recorded (baseline). After that, participants listened for 20 minutes via standard headphones to one of the same three audios as described in the first experiment. All were instructed to relax and listen with closed eyes in a comfortable position. After that, headphones were removed and participants were questioned about any unusual feeling during the course of the listening.

\subsection{Instrumentation}

\subsubsection{Stimulation}

20-min stimulation with sampling frequency of 44100 and 16 bits. The commercial audio consisted in 2 binaural beats on theta $(4 \mathrm{~Hz})$ and beta $(16 \mathrm{~Hz})$ at $200 \mathrm{~Hz}$ and $250 \mathrm{~Hz}$ carrier tones with $13 \mathrm{~dB}$ dynamic range; self- made audio consisted in 4 binaural beats in theta $(4 \mathrm{~Hz})$ at $100 \mathrm{~Hz}, 200 \mathrm{~Hz}, 250 \mathrm{~Hz}$, and $300 \mathrm{~Hz}$ carrier tones with another four binaural beats in beta $(16 \mathrm{~Hz})$ at $500 \mathrm{~Hz}, 650 \mathrm{~Hz}, 750 \mathrm{~Hz}$, and $900 \mathrm{~Hz}$ carrier tones with $18 \mathrm{~dB}$ dynamic range. We selected those particular frequencies of the binaural tones because we wanted to test the effects of commercial audio aimed at improving concentration and this worked for these frequencies binaural, both have pink noise because it is more comfortable to listen binaural beats, the placebo signal consist in the pink noise with $15 \mathrm{dBs}$ All developed with MATLAB.

Acoustic pressure levels of the applied acoustic stimuli are given in Table 1.

Table 1. Acoustic Pressure Levels of applied stimuli.

\begin{tabular}{|l|c|c|c|}
\hline & $\begin{array}{c}\text { Commercial } \\
\text { audio }\end{array}$ & $\begin{array}{c}\text { Self-made } \\
\text { audio }\end{array}$ & $\begin{array}{c}\text { Placebo } \\
\text { audio }\end{array}$ \\
\hline \multicolumn{4}{|c|}{ Channel L } \\
\hline Leq,1min & $83.2 \mathrm{~dB}$ & $78.3 \mathrm{~dB}$ & $83.2 \mathrm{~dB}$ \\
\hline LAeq,1min & $75.3 \mathrm{dBA}$ & $72.9 \mathrm{dBA}$ & $75.3 \mathrm{dBA}$ \\
\hline \multicolumn{4}{|c|}{ Channel R } \\
\hline Leq,1min & $84.6 \mathrm{~dB}$ & $80 \mathrm{~dB}$ & $84.6 \mathrm{~dB}$ \\
\hline LAeq,1min & $76 \mathrm{dBA}$ & $73 \mathrm{dBA}$ & $76 \mathrm{dBA}$ \\
\hline
\end{tabular}


For the application of sound we used Philips SHH9567 headphones.

\subsection{2. $E E G$}

EEG was recorded by a Brainvision Braimnamp EEG at 29 scalp points (International 10/20 system) with simultaneous registration of ECG during $20 \mathrm{~min}$. Sampling rate was $1000 \mathrm{~Hz}$. Mid-forehead electrode was the ground and Nuprep cream was placed on each electrode.

Spectral analysis of the EEG was calculated offline. A 30-second time interval free of artifacts was extracted from the baseline resting state and at 5,10 , 15, and 20 minutes of listening. The extracted intervals were notch-filtered at $50 \mathrm{~Hz}$ and band pass filtered between $1 \mathrm{~Hz}$ and $70 \mathrm{~Hz}$. EEG power was computed by FFT for the following frequency bands: delta (1-4 Hz), theta $(4-8 \mathrm{~Hz})$, alpha $(8-12 \mathrm{~Hz})$, beta $(12-$ $30 \mathrm{~Hz})$, gamma $(30-40 \mathrm{~Hz})$, and for two narrow bands $(0.2 \mathrm{~Hz}$ width $)$ centered at $4 \mathrm{~Hz}$ and $16 \mathrm{~Hz}$. Entrainment was defined for each band and electrode as the ratio of power between stimulation and pre-stimulation (baseline).

Therefore, we calculated four matrixes of $29 \times 7$ entrainment values for every subject in the experiment.

As the number of entrainment values was very high (29 electrodes $\times 7$ bands $\times 4$ moments), we considered results significant for $p<0.01$, in order to minimize type I errors. A non-parametric Kruskal-Wallis analysis of variance was performed.

\subsubsection{Perception test of differences}

The perception test of differences was developed to assess the speed and hits in partially ordered stimulation pattern similarities and differences. It is a discrimination test based on the similarities and differences principles. This type of testing have been shown positive correlations with general intelligence (REPARAZ et al., 1996).

The test consists of 60 graphic elements, in blocks of three elements each; the task is to determine which of the three faces is different from the other two.

\subsubsection{EMAV test}

The EMAV test assesses the attention capacity and effectiveness in children and adults. This test provides evidence on sustained attention in simple tasks of visual analysis and synthesis. It provides two levels of focus: Sustained Attention (AS) and Quality of Attention (CA) (PÉREZ, LAGO, 2000).

\subsubsection{Five Digit Test}

The Five Digit Test is a tool to evaluate cognitive processing speed, the ability to focus and refocus at- tention, and ability to cope with interference. Based on the known Stroop effect, but instead of using words and colors as stimulus, figures or digits are employed in this test, allowing for a greater variety of tasks and possibility to be used with less educated people, even those who do not know the language or cannot read (SEDÓ, 2004).

\section{Results}

Experiment A: There was no significant difference in scores between groups for any of the three tests (Table 2). A non-parametric Kruskal-Wallis analysis of variance was performed. Data are presented in Table 1 as the mean \pm s.d. $p<0.05$ was considered significant.

Table 2. Statistic analysis results.

\begin{tabular}{|l|c|c|c|c|}
\hline \multicolumn{1}{|c|}{ Test } & Placebo & $\begin{array}{c}\text { Binaural } \\
\text { commercial } \\
\text { group }\end{array}$ & $\begin{array}{c}\text { Binaural } \\
\text { experimental } \\
\text { group }\end{array}$ & $p$ \\
\hline \multicolumn{5}{|c|}{ Perception of differences } \\
\hline Score & $51.5 \pm 6.9$ & $49.6 \pm 6.6$ & $49.1 \pm 7.9$ & 0.52 \\
\hline \multicolumn{5}{|c|}{5 digit } \\
\hline $\begin{array}{l}\text { Reading } \\
\text { time }\end{array}$ & $19.6 \pm 3.5$ & $21.5 \pm 5.0$ & $19.4 \pm 3.6$ & 0.36 \\
\hline $\begin{array}{l}\text { Counting } \\
\text { time }\end{array}$ & $20.9 \pm 2.7$ & $21.9 \pm 3.3$ & $20.9 \pm 3.4$ & 0.38 \\
\hline $\begin{array}{l}\text { Election } \\
\text { time }\end{array}$ & $31.9 \pm 7.0$ & $31.3 \pm 4.5$ & $29.0 \pm 4.9$ & 0.43 \\
\hline $\begin{array}{l}\text { Alternation } \\
\text { time }\end{array}$ & $37.2 \pm 9.1$ & $38.6 \pm 5.0$ & $37.2 \pm 7.3$ & 0.23 \\
\hline \multicolumn{5}{|c|}{ EMAV-2 } \\
\hline Inhibition & $12.3 \pm 6.7$ & $9.8 \pm 5.5$ & $9.5 \pm 4.3$ & 0.54 \\
\hline Flexibility & $17.5 \pm 8.3$ & $17.1 \pm 5.5$ & $17.7 \pm 7.1$ & 0.77 \\
\hline
\end{tabular}

Experiment $\boldsymbol{B}$ : As the number of entrainment values was very high (29 electrodes $\times 7$ bands $\times 4$ moments), we considered results significant for $p<0.01$, in order to minimize type I errors. A non-parametric Kruskal-Wallis analysis of variance was performed but we could not find any significant differences between the three groups for any electrode, band, or moment of stimulation. The results of KruskalWallis analysis of variance are given for commercial audio (Tables 3-6), for self-made audio (Tables 7-10), and placebo audio (Tables 11-14).

- Commercial Audio,

- Self-made Audio,

- Placebo Audio. 
Table 3. Entrainment at $\mathbf{5} \mathbf{~ m i n}$ (Commercial Audio). Kruskal-Wallis p-significance.

\begin{tabular}{|c|c|c|c|c|c|c|c|}
\hline \multirow{2}{*}{ Electrode } & \multicolumn{7}{|c|}{ Bands } \\
\hline & Delta & Theta & Alpha & Beta & Gamma & Narrow $4 \mathrm{~Hz}$ & Narrow $14 \mathrm{~Hz}$ \\
\hline Fp1 & 0.03 & 0.46 & 0.03 & 0.17 & 0.46 & 0.60 & 0.60 \\
\hline Fp2 & 0.03 & 0.60 & 0.05 & 0.12 & 0.60 & 0.75 & 0.35 \\
\hline F3 & 0.17 & 0.46 & 0.05 & 0.07 & 0.60 & 0.17 & 0.46 \\
\hline $\mathrm{F} 4$ & 0.03 & 0.12 & 0.03 & 0.07 & 0.75 & 0.25 & 0.35 \\
\hline C3 & 0.07 & 0.17 & 0.05 & 0.17 & 0.75 & 0.03 & 0.60 \\
\hline $\mathrm{C} 4$ & 0.25 & 0.05 & 0.03 & 0.75 & 0.35 & 0.07 & 0.35 \\
\hline P3 & 0.03 & 0.07 & 0.03 & 0.60 & 0.05 & 0.03 & 0.35 \\
\hline P4 & 0.03 & 0.03 & 0.03 & 0.92 & 0.07 & 0.05 & 0.35 \\
\hline O1 & 0.03 & 0.05 & 0.03 & 0.75 & 0.05 & 0.05 & 0.03 \\
\hline $\mathrm{O} 2$ & 0.04 & 0.35 & 0.04 & 0.35 & 0.08 & 0.04 & 0.35 \\
\hline F7 & 0.17 & 0.60 & 0.03 & 0.25 & 0.60 & 0.03 & 0.75 \\
\hline F8 & 0.17 & 0.60 & 0.03 & 0.25 & 0.60 & 0.03 & 0.75 \\
\hline $\mathrm{T} 7$ & 0.17 & 0.46 & 0.03 & 0.46 & 0.46 & 0.03 & 0.75 \\
\hline T8 & 0.07 & 0.60 & 0.05 & 0.07 & 0.75 & 0.12 & 0.35 \\
\hline P7 & 0.03 & 0.25 & 0.03 & 0.92 & 0.05 & 0.03 & 0.07 \\
\hline P8 & 0.03 & 0.05 & 0.03 & 0.75 & 0.07 & 0.03 & 0.05 \\
\hline $\mathrm{Fz}$ & 0.25 & 0.12 & 0.05 & 0.35 & 0.46 & 0.60 & 0.75 \\
\hline $\mathrm{Cz}$ & 0.12 & 0.75 & 0.03 & 0.60 & 0.46 & 0.46 & 0.60 \\
\hline $\mathrm{Pz}$ & 0.03 & 0.07 & 0.03 & 0.17 & 0.05 & 0.03 & 0.75 \\
\hline $\mathrm{FC} 1$ & 0.75 & 0.35 & 0.03 & 0.92 & 0.60 & 0.07 & 0.46 \\
\hline $\mathrm{FC} 2$ & 0.75 & 0.05 & 0.03 & 0.75 & 0.75 & 0.03 & 0.92 \\
\hline CP1 & 0.05 & 0.25 & 0.03 & 0.12 & 0.07 & 0.03 & 0.60 \\
\hline CP2 & 0.05 & 0.03 & 0.03 & 0.35 & 0.07 & 0.12 & 0.35 \\
\hline FC5 & 0.12 & 0.17 & 0.03 & 0.60 & 0.46 & 0.03 & 0.92 \\
\hline FC6 & 0.46 & 0.92 & 0.03 & 0.25 & 0.46 & 0.17 & 0.35 \\
\hline CP5 & 0.07 & 0.46 & 0.03 & 0.75 & 0.05 & 0.03 & 0.60 \\
\hline CP6 & 0.07 & 0.46 & 0.03 & 0.75 & 0.05 & 0.03 & 0.60 \\
\hline TP9 & 0.05 & 0.60 & 0.03 & 0.12 & 0.92 & 0.03 & 0.60 \\
\hline TP10 & 0.05 & 0.92 & 0.03 & 0.03 & 0.92 & 0.07 & 0.92 \\
\hline
\end{tabular}

Table 4. Entrainment at $\mathbf{1 0} \mathbf{~ m i n}$ (Commercial Audio). Kruskal-Wallis p-significance.

\begin{tabular}{|c|c|c|c|c|c|c|c|}
\hline \multirow{2}{*}{ Electrode } & \multicolumn{7}{|c|}{ Bands } \\
\hline & Delta & Theta & Alpha & Beta & Gamma & Narrow $4 \mathrm{~Hz}$ & Narrow $14 \mathrm{~Hz}$ \\
\hline Fp1 & 0.92 & 0.12 & 0.17 & 0.75 & 0.25 & 0.46 & 0.46 \\
\hline Fp2 & 0.92 & 0.35 & 0.12 & 0.46 & 0.25 & 0.75 & 0.92 \\
\hline F3 & 0.75 & 0.92 & 0.17 & 0.75 & 0.35 & 0.75 & 0.92 \\
\hline $\mathrm{F} 4$ & 0.46 & 0.12 & 0.12 & 0.75 & 0.35 & 0.92 & 0.75 \\
\hline C3 & 0.46 & 0.12 & 0.05 & 0.60 & 0.46 & 0.07 & 0.25 \\
\hline $\mathrm{C} 4$ & 0.46 & 0.03 & 0.07 & 0.03 & 0.46 & 0.46 & 0.03 \\
\hline P3 & 0.17 & 0.05 & 0.03 & 0.35 & 0.12 & 0.05 & 0.17 \\
\hline P4 & 0.07 & 0.05 & 0.03 & 0.60 & 0.12 & 0.12 & 0.75 \\
\hline $\mathrm{O} 1$ & 0.05 & 0.03 & 0.03 & 0.75 & 0.07 & 0.03 & 0.46 \\
\hline $\mathrm{O} 2$ & 0.08 & 0.08 & 0.08 & 0.69 & 0.14 & 0.04 & 0.35 \\
\hline F7 & 0.75 & 0.46 & 0.12 & 0.60 & 0.35 & 0.75 & 0.92 \\
\hline F8 & 0.75 & 0.46 & 0.12 & 0.60 & 0.35 & 0.75 & 0.92 \\
\hline $\mathrm{T} 7$ & 0.75 & 0.46 & 0.07 & 0.46 & 0.60 & 0.35 & 0.46 \\
\hline $\mathrm{T} 8$ & 0.35 & 0.17 & 0.05 & 0.75 & 0.92 & 0.75 & 0.75 \\
\hline $\mathrm{P} 7$ & 0.12 & 0.12 & 0.03 & 0.92 & 0.12 & 0.07 & 0.46 \\
\hline P8 & 0.25 & 0.05 & 0.03 & 0.92 & 0.07 & 0.05 & 0.46 \\
\hline $\mathrm{Fz}$ & 0.75 & 0.75 & 0.35 & 0.92 & 0.75 & 0.35 & 0.60 \\
\hline $\mathrm{Cz}$ & 0.25 & 0.05 & 0.12 & 0.75 & 0.35 & 0.60 & 0.60 \\
\hline $\mathrm{Pz}$ & 0.35 & 0.05 & 0.03 & 0.46 & 0.05 & 0.03 & 0.35 \\
\hline FC1 & 0.60 & 0.05 & 0.12 & 0.03 & 0.60 & 0.05 & 0.07 \\
\hline $\mathrm{FC} 2$ & 0.60 & 0.05 & 0.12 & 0.07 & 0.60 & 0.92 & 0.17 \\
\hline CP1 & 0.35 & 0.12 & 0.05 & 0.35 & 0.17 & 0.03 & 0.12 \\
\hline CP2 & 0.60 & 0.05 & 0.05 & 0.35 & 0.12 & 0.92 & 0.46 \\
\hline FC5 & 0.75 & 0.35 & 0.12 & 0.92 & 0.35 & 0.25 & 0.25 \\
\hline FC6 & 0.92 & 0.46 & 0.03 & 0.92 & 0.75 & 0.35 & 0.75 \\
\hline CP5 & 0.17 & 0.46 & 0.03 & 0.75 & 0.17 & 0.25 & 0.46 \\
\hline CP6 & 0.17 & 0.46 & 0.03 & 0.75 & 0.17 & 0.25 & 0.46 \\
\hline TP9 & 0.17 & 0.12 & 0.03 & 0.25 & 0.46 & 0.25 & 0.46 \\
\hline TP10 & 0.25 & 0.12 & 0.03 & 0.35 & 0.12 & 0.12 & 0.46 \\
\hline
\end{tabular}


Table 5. Entrainment at $\mathbf{1 5}$ min (Commercial Audio). Kruskal-Wallis p-significance.

\begin{tabular}{|c|c|c|c|c|c|c|c|}
\hline \multirow{2}{*}{ Electrode } & \multicolumn{7}{|c|}{ Bands } \\
\hline & Delta & Theta & Alpha & Beta & Gamma & Narrow $4 \mathrm{~Hz}$ & Narrow $14 \mathrm{~Hz}$ \\
\hline Fp1 & 0.46 & 0.12 & 0.17 & 0.75 & 0.07 & 0.46 & 0.35 \\
\hline Fp2 & 0.25 & 0.07 & 0.17 & 0.92 & 0.12 & 0.92 & 0.60 \\
\hline F3 & 0.75 & 0.46 & 0.07 & 0.92 & 0.46 & 0.75 & 0.60 \\
\hline F4 & 0.75 & 0.75 & 0.03 & 0.92 & 0.25 & 0.75 & 0.46 \\
\hline C3 & 0.17 & 0.03 & 0.03 & 0.46 & 0.92 & 0.05 & 0.35 \\
\hline $\mathrm{C} 4$ & 0.46 & 0.05 & 0.05 & 0.46 & 0.60 & 0.05 & 0.46 \\
\hline P3 & 0.03 & 0.05 & 0.03 & 0.92 & 0.17 & 0.12 & 0.05 \\
\hline $\mathrm{P} 4$ & 0.03 & 0.03 & 0.03 & 0.92 & 0.25 & 0.07 & 0.46 \\
\hline O1 & 0.05 & 0.03 & 0.05 & 0.92 & 0.12 & 0.12 & 0.03 \\
\hline $\mathrm{O} 2$ & 0.08 & 0.08 & 0.08 & 0.69 & 0.14 & 0.14 & 0.89 \\
\hline F7 & 0.60 & 0.25 & 0.12 & 0.35 & 0.75 & 0.60 & 0.60 \\
\hline F8 & 0.60 & 0.25 & 0.12 & 0.35 & 0.75 & 0.60 & 0.60 \\
\hline $\mathrm{T} 7$ & 0.25 & 0.05 & 0.07 & 0.35 & 0.92 & 0.17 & 0.75 \\
\hline T8 & 0.25 & 0.07 & 0.12 & 0.75 & 0.75 & 0.05 & 0.46 \\
\hline P7 & 0.07 & 0.07 & 0.05 & 0.92 & 0.12 & 0.07 & 0.12 \\
\hline P8 & 0.05 & 0.05 & 0.05 & 0.60 & 0.12 & 0.05 & 0.92 \\
\hline $\mathrm{Fz}$ & 0.60 & 0.60 & 0.05 & 0.46 & 0.75 & 0.75 & 0.35 \\
\hline $\mathrm{Cz}$ & 0.25 & 0.05 & 0.05 & 0.17 & 0.92 & 0.46 & 0.92 \\
\hline $\mathrm{Pz}$ & 0.03 & 0.03 & 0.03 & 0.25 & 0.12 & 0.07 & 0.35 \\
\hline $\mathrm{FC} 1$ & 0.92 & 0.07 & 0.05 & 0.46 & 0.07 & 0.03 & 0.92 \\
\hline $\mathrm{FC} 2$ & 0.75 & 0.35 & 0.07 & 0.92 & 0.75 & 0.25 & 0.75 \\
\hline CP1 & 0.12 & 0.03 & 0.03 & 0.60 & 0.07 & 0.12 & 0.05 \\
\hline $\mathrm{CP} 2$ & 0.07 & 0.03 & 0.03 & 0.12 & 0.46 & 0.12 & 0.35 \\
\hline FC5 & 0.25 & 0.46 & 0.05 & 0.46 & 0.25 & 0.35 & 0.92 \\
\hline FC6 & 0.46 & 0.35 & 0.03 & 0.75 & 0.46 & 0.75 & 0.25 \\
\hline CP5 & 0.05 & 0.05 & 0.03 & 0.75 & 0.07 & 0.12 & 0.12 \\
\hline CP6 & 0.05 & 0.05 & 0.03 & 0.75 & 0.07 & 0.12 & 0.12 \\
\hline TP9 & 0.12 & 0.35 & 0.03 & 0.35 & 0.07 & 0.12 & 0.92 \\
\hline TP10 & 0.25 & 0.05 & 0.03 & 0.35 & 0.35 & 0.05 & 0.75 \\
\hline
\end{tabular}

Table 6. Entrainment at $\mathbf{2 0}$ min / final (Commercial Audio). Kruskal-Wallis p-significance.

\begin{tabular}{|c|c|c|c|c|c|c|c|}
\hline \multirow{2}{*}{ Electrode } & \multicolumn{7}{|c|}{ Bands } \\
\hline & Delta & Theta & Alpha & Beta & Gamma & Narrow $4 \mathrm{~Hz}$ & Narrow $14 \mathrm{~Hz}$ \\
\hline Fp1 & 0.92 & 0.75 & 0.25 & 0.75 & 0.75 & 0.92 & 0.46 \\
\hline Fp2 & 0.92 & 0.75 & 0.17 & 0.75 & 0.75 & 0.75 & 0.92 \\
\hline F3 & 0.75 & 0.60 & 0.60 & 0.25 & 0.92 & 0.92 & 0.07 \\
\hline $\mathrm{F} 4$ & 0.75 & 0.35 & 0.25 & 0.92 & 0.46 & 0.92 & 0.17 \\
\hline C3 & 0.92 & 0.03 & 0.46 & 0.92 & 0.75 & 0.25 & 0.35 \\
\hline $\mathrm{C} 4$ & 0.75 & 0.03 & 0.25 & 0.35 & 0.92 & 0.92 & 0.46 \\
\hline P3 & 0.05 & 0.17 & 0.12 & 0.46 & 0.75 & 0.12 & 0.17 \\
\hline P4 & 0.07 & 0.07 & 0.12 & 0.25 & 0.92 & 0.12 & 0.75 \\
\hline $\mathrm{O} 1$ & 0.07 & 0.12 & 0.12 & 0.12 & 0.60 & 0.12 & 0.60 \\
\hline $\mathrm{O} 2$ & 0.08 & 0.08 & 0.14 & 0.08 & 0.69 & 0.08 & 0.50 \\
\hline F7 & 0.92 & 0.92 & 0.25 & 0.92 & 0.17 & 0.35 & 0.07 \\
\hline F8 & 0.92 & 0.92 & 0.25 & 0.92 & 0.17 & 0.35 & 0.07 \\
\hline $\mathrm{T} 7$ & 0.60 & 0.46 & 0.17 & 0.60 & 0.60 & 0.03 & 0.35 \\
\hline $\mathrm{T} 8$ & 0.46 & 0.25 & 0.46 & 0.75 & 0.92 & 0.46 & 0.46 \\
\hline $\mathrm{P} 7$ & 0.07 & 0.12 & 0.17 & 0.17 & 0.46 & 0.07 & 0.92 \\
\hline P8 & 0.17 & 0.05 & 0.12 & 0.46 & 0.60 & 0.12 & 0.75 \\
\hline $\mathrm{Fz}$ & 0.75 & 0.92 & 0.35 & 0.60 & 0.75 & 0.60 & 0.35 \\
\hline $\mathrm{Cz}$ & 0.75 & 0.17 & 0.12 & 0.25 & 0.92 & 0.46 & 0.25 \\
\hline $\mathrm{Pz}$ & 0.05 & 0.03 & 0.07 & 0.35 & 0.46 & 0.05 & 0.35 \\
\hline FC1 & 0.35 & 0.03 & 0.60 & 0.05 & 0.60 & 0.03 & 0.03 \\
\hline $\mathrm{FC} 2$ & 0.35 & 0.92 & 0.12 & 0.75 & 0.46 & 0.92 & 0.35 \\
\hline CP1 & 0.46 & 0.12 & 0.05 & 0.92 & 0.92 & 0.12 & 0.25 \\
\hline CP2 & 0.17 & 0.05 & 0.12 & 0.92 & 0.75 & 0.12 & 0.75 \\
\hline FC5 & 0.75 & 0.75 & 0.35 & 0.92 & 0.92 & 0.17 & 0.35 \\
\hline FC6 & 0.75 & 0.92 & 0.35 & 0.92 & 0.92 & 0.92 & 0.75 \\
\hline CP5 & 0.25 & 0.25 & 0.17 & 0.92 & 0.60 & 0.12 & 0.92 \\
\hline CP6 & 0.25 & 0.25 & 0.17 & 0.92 & 0.60 & 0.12 & 0.92 \\
\hline TP9 & 0.17 & 0.35 & 0.05 & 0.17 & 0.46 & 0.17 & 0.25 \\
\hline TP10 & 0.12 & 0.25 & 0.03 & 0.75 & 0.75 & 0.12 & 0.60 \\
\hline
\end{tabular}


Table 7. Entrainment at $\mathbf{5}$ min (Self-made Audio). Kruskal-Wallis $p$-significance.

\begin{tabular}{|c|c|c|c|c|c|c|c|}
\hline \multirow{2}{*}{ Electrode } & \multicolumn{7}{|c|}{ Bands } \\
\hline & Delta & Theta & Alpha & Beta & Gamma & Narrow $4 \mathrm{~Hz}$ & Narrow $14 \mathrm{~Hz}$ \\
\hline Fp1 & 0.75 & 0.92 & 0.46 & 0.46 & 0.05 & 0.60 & 0.60 \\
\hline Fp2 & 0.75 & 0.75 & 0.46 & 0.75 & 0.12 & 0.75 & 0.46 \\
\hline F3 & 0.75 & 0.25 & 0.92 & 0.03 & 0.25 & 0.75 & 0.17 \\
\hline $\mathrm{F} 4$ & 0.25 & 0.17 & 0.92 & 0.12 & 0.03 & 0.92 & 0.35 \\
\hline C3 & 0.60 & 0.92 & 0.60 & 0.46 & 0.35 & 0.03 & 0.12 \\
\hline $\mathrm{C} 4$ & 0.35 & 0.75 & 0.60 & 0.25 & 0.35 & 0.25 & 0.92 \\
\hline P3 & 0.03 & 0.12 & 0.07 & 0.46 & 0.05 & 0.35 & 0.17 \\
\hline P4 & 0.07 & 0.60 & 0.46 & 0.75 & 0.05 & 0.92 & 0.60 \\
\hline O1 & 0.12 & 0.35 & 0.05 & 0.75 & 0.17 & 0.35 & 0.75 \\
\hline $\mathrm{O} 2$ & 0.35 & 0.69 & 0.89 & 0.35 & 0.35 & 0.69 & 0.14 \\
\hline F7 & 0.46 & 0.92 & 0.46 & 0.35 & 0.35 & 0.75 & 0.35 \\
\hline F8 & 0.46 & 0.92 & 0.46 & 0.35 & 0.35 & 0.75 & 0.35 \\
\hline $\mathrm{T} 7$ & 0.75 & 0.60 & 0.46 & 0.60 & 0.92 & 0.35 & 0.25 \\
\hline T8 & 0.75 & 0.46 & 0.60 & 0.92 & 0.60 & 0.92 & 0.92 \\
\hline P7 & 0.35 & 0.46 & 0.25 & 0.92 & 0.17 & 0.46 & 0.46 \\
\hline P8 & 0.35 & 0.92 & 0.92 & 0.46 & 0.17 & 0.92 & 0.60 \\
\hline $\mathrm{Fz}$ & 0.46 & 0.17 & 0.46 & 0.05 & 0.03 & 0.60 & 0.35 \\
\hline $\mathrm{Cz}$ & 0.92 & 0.35 & 0.35 & 0.17 & 0.03 & 0.35 & 0.17 \\
\hline $\mathrm{Pz}$ & 0.12 & 0.35 & 0.25 & 0.46 & 0.17 & 0.46 & 0.25 \\
\hline $\mathrm{FC} 1$ & 0.35 & 0.92 & 0.46 & 0.03 & 0.25 & 0.03 & 0.25 \\
\hline $\mathrm{FC} 2$ & 0.12 & 0.12 & 0.46 & 0.17 & 0.05 & 0.05 & 0.75 \\
\hline CP1 & 0.75 & 0.17 & 0.25 & 0.46 & 0.07 & 0.75 & 0.25 \\
\hline CP2 & 0.60 & 0.46 & 0.35 & 0.75 & 0.05 & 0.92 & 0.75 \\
\hline FC5 & 0.35 & 0.75 & 0.60 & 0.25 & 0.25 & 0.05 & 0.46 \\
\hline FC6 & 0.35 & 0.46 & 0.92 & 0.75 & 0.92 & 0.12 & 0.60 \\
\hline CP5 & 0.35 & 0.35 & 0.25 & 0.92 & 0.17 & 0.46 & 0.35 \\
\hline CP6 & 0.35 & 0.35 & 0.25 & 0.92 & 0.17 & 0.46 & 0.35 \\
\hline TP9 & 0.92 & 0.60 & 0.60 & 0.92 & 0.07 & 0.46 & 0.46 \\
\hline TP10 & 0.46 & 0.60 & 0.60 & 0.75 & 0.60 & 0.75 & 0.75 \\
\hline
\end{tabular}

Table 8. Entrainment at $\mathbf{1 0}$ min (Self-made Audio). Kruskal-Wallis p-significance.

\begin{tabular}{|c|c|c|c|c|c|c|c|}
\hline \multirow{2}{*}{ Electrode } & \multicolumn{7}{|c|}{ Bands } \\
\hline & Delta & Theta & Alpha & Beta & Gamma & Narrow $4 \mathrm{~Hz}$ & Narrow $14 \mathrm{~Hz}$ \\
\hline Fp1 & 0.92 & 0.75 & 0.92 & 0.46 & 0.03 & 0.35 & 0.17 \\
\hline Fp2 & 0.60 & 0.92 & 0.75 & 0.17 & 0.12 & 0.46 & 0.35 \\
\hline F3 & 0.60 & 0.92 & 0.92 & 0.60 & 0.12 & 0.60 & 0.60 \\
\hline $\mathrm{F} 4$ & 0.25 & 0.92 & 0.75 & 0.12 & 0.03 & 0.92 & 0.46 \\
\hline C3 & 0.46 & 0.75 & 0.17 & 0.92 & 0.75 & 0.75 & 0.25 \\
\hline $\mathrm{C} 4$ & 0.75 & 0.60 & 0.92 & 0.75 & 0.07 & 0.35 & 0.60 \\
\hline P3 & 0.12 & 0.60 & 0.75 & 0.92 & 0.05 & 0.75 & 0.46 \\
\hline P4 & 0.35 & 0.46 & 0.60 & 0.46 & 0.12 & 0.75 & 0.60 \\
\hline O1 & 0.05 & 0.35 & 0.03 & 0.92 & 0.05 & 0.60 & 0.75 \\
\hline $\mathrm{O} 2$ & 0.08 & 0.50 & 0.14 & 0.50 & 0.22 & 0.50 & 0.50 \\
\hline F7 & 0.92 & 0.92 & 0.25 & 0.92 & 0.92 & 0.35 & 0.92 \\
\hline F8 & 0.92 & 0.92 & 0.25 & 0.92 & 0.92 & 0.35 & 0.92 \\
\hline T7 & 0.12 & 0.60 & 0.60 & 0.92 & 0.92 & 0.46 & 0.75 \\
\hline T8 & 0.17 & 0.75 & 0.92 & 0.60 & 0.92 & 0.75 & 0.92 \\
\hline P7 & 0.12 & 0.25 & 0.05 & 0.46 & 0.05 & 0.35 & 0.75 \\
\hline P8 & 0.17 & 0.46 & 0.35 & 0.60 & 0.05 & 0.75 & 0.92 \\
\hline $\mathrm{Fz}$ & 0.25 & 0.75 & 0.75 & 0.25 & 0.03 & 0.60 & 0.25 \\
\hline $\mathrm{Cz}$ & 0.92 & 0.60 & 0.75 & 0.60 & 0.05 & 0.25 & 0.75 \\
\hline $\mathrm{Pz}$ & 0.25 & 0.75 & 0.92 & 0.60 & 0.25 & 0.75 & 0.92 \\
\hline FC1 & 0.75 & 0.60 & 0.92 & 0.75 & 0.25 & 0.25 & 0.75 \\
\hline $\mathrm{FC} 2$ & 0.35 & 0.46 & 0.60 & 0.60 & 0.05 & 0.25 & 0.92 \\
\hline $\mathrm{CP} 1$ & 0.92 & 0.75 & 0.92 & 0.75 & 0.17 & 0.75 & 0.75 \\
\hline CP2 & 0.75 & 0.60 & 0.92 & 0.60 & 0.12 & 0.60 & 0.92 \\
\hline FC5 & 0.60 & 0.46 & 0.25 & 0.05 & 0.35 & 0.60 & 0.25 \\
\hline FC6 & 0.35 & 0.92 & 0.60 & 0.92 & 0.25 & 0.92 & 0.92 \\
\hline CP5 & 0.12 & 0.60 & 0.12 & 0.92 & 0.05 & 0.75 & 0.46 \\
\hline CP6 & 0.12 & 0.60 & 0.12 & 0.92 & 0.05 & 0.75 & 0.46 \\
\hline TP9 & 0.07 & 0.60 & 0.12 & 0.75 & 0.25 & 0.75 & 0.46 \\
\hline TP10 & 0.25 & 0.92 & 0.35 & 0.60 & 0.60 & 0.60 & 0.35 \\
\hline
\end{tabular}


Table 9. Entrainment at $\mathbf{1 5} \mathbf{~ m i n}$ (Self-made Audio). Kruskal-Wallis $p$-significance.

\begin{tabular}{|l|c|c|c|c|c|c|c|}
\hline \multirow{2}{*}{ Electrode } & \multicolumn{9}{|c|}{ Bands } \\
\cline { 2 - 7 } & Delta & Theta & Alpha & Beta & Gamma & Narrow 4 Hz & Narrow 14 Hz \\
\hline Fp1 & 0.92 & 0.17 & 0.07 & 0.17 & 0.07 & 0.75 & 0.35 \\
Fp2 & 0.60 & 0.35 & 0.25 & 0.17 & 0.25 & 0.60 & 0.60 \\
F3 & 0.92 & 0.46 & 0.05 & 0.12 & 0.46 & 0.92 & 0.35 \\
F4 & 0.75 & 0.92 & 0.12 & 0.25 & 0.75 & 0.60 & 0.92 \\
C3 & 0.03 & 0.60 & 0.03 & 0.35 & 0.92 & 0.35 & 0.60 \\
C4 & 0.25 & 0.60 & 0.03 & 0.46 & 0.12 & 0.60 & 0.46 \\
P3 & 0.12 & 0.25 & 0.12 & 0.35 & 0.03 & 0.17 & 0.46 \\
P4 & 0.12 & 0.12 & 0.12 & 0.75 & 0.03 & 0.12 & 0.92 \\
O1 & 0.03 & 0.12 & 0.03 & 0.35 & 0.03 & 0.12 & 0.75 \\
O2 & 0.22 & 0.22 & 0.08 & 0.89 & 0.08 & 0.22 & 0.69 \\
F7 & 0.60 & 0.35 & 0.12 & 0.25 & 0.92 & 0.17 & 0.46 \\
F8 & 0.60 & 0.35 & 0.12 & 0.25 & 0.92 & 0.17 & 0.46 \\
T7 & 0.17 & 0.92 & 0.25 & 0.35 & 0.92 & 0.12 & 0.75 \\
T8 & 0.05 & 0.75 & 0.46 & 0.46 & 0.60 & 0.17 & 0.35 \\
P7 & 0.05 & 0.17 & 0.05 & 0.60 & 0.12 & 0.17 & 0.92 \\
P8 & 0.12 & 0.35 & 0.12 & 0.92 & 0.07 & 0.12 & 0.75 \\
Fz & 0.75 & 0.46 & 0.12 & 0.17 & 0.12 & 0.75 & 0.60 \\
Cz & 0.07 & 0.60 & 0.12 & 0.35 & 0.05 & 0.92 & 0.07 \\
Pz & 0.12 & 0.12 & 0.25 & 0.35 & 0.03 & 0.12 & 0.35 \\
FC1 & 0.92 & 0.35 & 0.07 & 0.07 & 0.75 & 0.60 & 0.17 \\
FC2 & 0.75 & 0.35 & 0.05 & 0.12 & 0.60 & 0.92 & 0.75 \\
CP1 & 0.12 & 0.60 & 0.12 & 0.35 & 0.12 & 0.25 & 0.12 \\
CP2 & 0.12 & 0.12 & 0.05 & 0.46 & 0.03 & 0.17 & 0.17 \\
FC5 & 0.25 & 0.60 & 0.05 & 0.25 & 0.92 & 0.75 & 0.17 \\
FC6 & 0.12 & 0.75 & 0.05 & 0.35 & 0.25 & 0.75 & 0.92 \\
CP5 & 0.12 & 0.60 & 0.07 & 0.46 & 0.17 & 0.35 & 0.75 \\
CP6 & 0.12 & 0.60 & 0.07 & 0.46 & 0.17 & 0.35 & 0.75 \\
TP9 & 0.05 & 0.35 & 0.05 & 0.75 & 0.17 & 0.25 & 0.46 \\
TP10 & 0.07 & 0.60 & 0.12 & 0.35 & 0.92 & 0.35 & 0.35 \\
\hline
\end{tabular}

Table 10. Entrainment at $\mathbf{2 0}$ min / final (Self-made Audio). Kruskal-Wallis $p$-significance.

\begin{tabular}{|l|c|c|c|c|c|c|c|}
\hline \multirow{2}{*}{ Electrode } & \multicolumn{9}{|c|}{ Bands } \\
\cline { 2 - 7 } & Delta & Theta & Alpha & Beta & Gamma & Narrow 4 Hz & Narrow 14 Hz \\
\hline Fp1 & 0.25 & 0.12 & 0.12 & 0.92 & 0.03 & 0.92 & 0.75 \\
Fp2 & 0.35 & 0.46 & 0.17 & 0.60 & 0.46 & 0.60 & 0.75 \\
F3 & 0.75 & 0.35 & 0.25 & 0.35 & 0.07 & 0.46 & 0.25 \\
F4 & 0.35 & 0.92 & 0.35 & 0.17 & 0.03 & 0.60 & 0.46 \\
C3 & 0.35 & 0.35 & 0.25 & 0.75 & 0.46 & 0.92 & 0.05 \\
C4 & 0.25 & 0.92 & 0.35 & 0.35 & 0.03 & 0.60 & 0.75 \\
P3 & 0.12 & 0.46 & 0.25 & 0.35 & 0.05 & 0.25 & 0.03 \\
P4 & 0.12 & 0.25 & 0.25 & 0.75 & 0.03 & 0.25 & 0.25 \\
O1 & 0.12 & 0.25 & 0.03 & 0.35 & 0.03 & 0.12 & 0.03 \\
O2 & 0.14 & 0.22 & 0.08 & 0.69 & 0.22 & 0.22 & 0.69 \\
F7 & 0.17 & 0.25 & 0.17 & 0.46 & 0.60 & 0.46 & 0.46 \\
F8 & 0.17 & 0.25 & 0.17 & 0.46 & 0.60 & 0.46 & 0.46 \\
T7 & 0.60 & 0.25 & 0.46 & 0.75 & 0.75 & 0.35 & 0.35 \\
T8 & 0.17 & 0.92 & 0.60 & 0.46 & 0.60 & 0.60 & 0.92 \\
P7 & 0.46 & 0.92 & 0.12 & 0.92 & 0.03 & 0.35 & 0.25 \\
P8 & 0.07 & 0.35 & 0.17 & 0.92 & 0.03 & 0.25 & 0.75 \\
Fz & 0.35 & 0.35 & 0.12 & 0.46 & 0.25 & 0.92 & 0.46 \\
Cz & 0.35 & 0.60 & 0.12 & 0.46 & 0.25 & 0.35 & 0.12 \\
Pz & 0.17 & 0.25 & 0.25 & 0.35 & 0.03 & 0.17 & 0.05 \\
FC1 & 0.25 & 0.35 & 0.35 & 0.46 & 0.75 & 0.35 & 0.35 \\
FC2 & 0.75 & 0.92 & 0.25 & 0.60 & 0.25 & 0.92 & 0.60 \\
CP1 & 0.35 & 0.75 & 0.35 & 0.75 & 0.05 & 0.25 & 0.03 \\
CP2 & 0.35 & 0.46 & 0.35 & 0.75 & 0.05 & 0.25 & 0.17 \\
FC5 & 0.46 & 0.60 & 0.17 & 0.12 & 0.60 & 0.75 & 0.03 \\
FC6 & 0.12 & 0.46 & 0.35 & 0.60 & 0.25 & 0.92 & 0.60 \\
CP5 & 0.25 & 0.60 & 0.12 & 0.46 & 0.03 & 0.60 & 0.12 \\
CP6 & 0.25 & 0.60 & 0.12 & 0.46 & 0.03 & 0.60 & 0.12 \\
TP9 & 0.12 & 0.25 & 0.17 & 0.35 & 0.60 & 0.12 & 0.07 \\
TP10 & 0.07 & 0.92 & 0.12 & 0.35 & 0.35 & 0.25 & 0.75 \\
\hline
\end{tabular}


Table 11. Entrainment at $\mathbf{5} \mathbf{~ m i n}$ (Placebo audio). Kruskal-Wallis $p$-significance.

\begin{tabular}{|c|c|c|c|c|c|c|c|}
\hline \multirow{2}{*}{ Electrode } & \multicolumn{7}{|c|}{ Bands } \\
\hline & Delta & Theta & Alpha & Beta & Gamma & Narrow $4 \mathrm{~Hz}$ & Narrow $14 \mathrm{~Hz}$ \\
\hline Fp1 & 0.17 & 0.05 & 0.35 & 0.25 & 0.25 & 0.17 & 0.35 \\
\hline Fp2 & 0.25 & 0.35 & 0.60 & 0.25 & 0.35 & 0.12 & 0.25 \\
\hline F3 & 0.25 & 0.12 & 0.92 & 0.46 & 0.92 & 0.60 & 0.25 \\
\hline F4 & 0.92 & 0.25 & 0.92 & 0.25 & 0.92 & 0.25 & 0.60 \\
\hline C3 & 0.60 & 0.12 & 0.92 & 0.25 & 0.60 & 0.17 & 0.46 \\
\hline $\mathrm{C} 4$ & 0.60 & 0.07 & 0.46 & 0.92 & 0.60 & 0.46 & 0.46 \\
\hline P3 & 0.92 & 0.46 & 0.60 & 0.46 & 0.92 & 0.75 & 0.46 \\
\hline $\mathrm{P} 4$ & 0.46 & 0.35 & 0.35 & 0.46 & 0.60 & 0.46 & 0.75 \\
\hline O1 & 0.60 & 0.75 & 0.92 & 0.60 & 0.46 & 0.46 & 0.75 \\
\hline $\mathrm{O} 2$ & 0.89 & 1.00 & 1.00 & 0.78 & 0.48 & 0.48 & 0.05 \\
\hline F7 & 0.35 & 0.07 & 0.75 & 0.46 & 0.75 & 0.25 & 0.46 \\
\hline F8 & 0.35 & 0.07 & 0.75 & 0.46 & 0.75 & 0.25 & 0.46 \\
\hline $\mathrm{T} 7$ & 0.60 & 0.60 & 0.46 & 0.35 & 0.75 & 0.75 & 0.05 \\
\hline T8 & 0.92 & 0.12 & 0.60 & 0.60 & 0.07 & 0.75 & 0.92 \\
\hline P7 & 0.75 & 0.92 & 0.75 & 0.07 & 0.75 & 0.92 & 0.75 \\
\hline P8 & 0.60 & 0.46 & 0.35 & 0.92 & 0.60 & 0.75 & 0.35 \\
\hline $\mathrm{Fz}$ & 0.25 & 0.46 & 0.46 & 0.25 & 0.35 & 0.92 & 0.35 \\
\hline $\mathrm{Cz}$ & 0.60 & 0.60 & 0.35 & 0.25 & 0.46 & 0.25 & 0.35 \\
\hline $\mathrm{Pz}$ & 0.75 & 0.60 & 0.35 & 0.35 & 0.46 & 0.60 & 0.92 \\
\hline FC1 & 0.60 & 0.25 & 0.17 & 0.75 & 0.75 & 0.35 & 0.35 \\
\hline $\mathrm{FC} 2$ & 0.75 & 0.60 & 0.75 & 0.46 & 0.25 & 0.46 & 0.46 \\
\hline CP1 & 0.35 & 0.46 & 0.25 & 0.60 & 0.92 & 0.12 & 0.46 \\
\hline CP2 & 0.35 & 0.60 & 0.35 & 0.75 & 0.75 & 0.25 & 0.75 \\
\hline FC5 & 0.60 & 0.35 & 0.75 & 0.60 & 0.35 & 0.60 & 0.60 \\
\hline FC6 & 0.92 & 0.07 & 0.92 & 0.25 & 0.17 & 0.60 & 0.75 \\
\hline CP5 & 0.46 & 0.12 & 0.35 & 0.46 & 0.25 & 0.35 & 0.60 \\
\hline CP6 & 0.46 & 0.12 & 0.35 & 0.46 & 0.25 & 0.35 & 0.60 \\
\hline TP9 & 0.75 & 0.60 & 0.92 & 0.03 & 0.92 & 0.60 & 0.60 \\
\hline TP10 & 0.75 & 0.12 & 0.92 & 0.46 & 0.60 & 0.75 & 0.75 \\
\hline
\end{tabular}

Table 12. Entrainment at $\mathbf{1 0} \mathbf{~ m i n}$ (Placebo audio). Kruskal-Wallis p-significance.

\begin{tabular}{|c|c|c|c|c|c|c|c|}
\hline \multirow{2}{*}{ Electrode } & \multicolumn{7}{|c|}{ Bands } \\
\hline & Delta & Theta & Alpha & Beta & Gamma & Narrow $4 \mathrm{~Hz}$ & Narrow $14 \mathrm{~Hz}$ \\
\hline Fp1 & 0.07 & 0.07 & 0.60 & 0.35 & 0.35 & 0.07 & 0.25 \\
\hline Fp2 & 0.05 & 0.05 & 0.35 & 0.05 & 0.17 & 0.05 & 0.35 \\
\hline F3 & 0.35 & 0.25 & 0.75 & 0.25 & 0.60 & 0.12 & 0.60 \\
\hline $\mathrm{F} 4$ & 0.46 & 0.35 & 0.75 & 0.92 & 0.46 & 0.25 & 0.92 \\
\hline C3 & 0.92 & 0.25 & 0.46 & 0.46 & 0.75 & 0.60 & 0.25 \\
\hline $\mathrm{C} 4$ & 0.92 & 0.12 & 0.46 & 0.75 & 0.92 & 0.75 & 0.25 \\
\hline P3 & 0.46 & 0.92 & 0.35 & 0.92 & 0.92 & 0.75 & 0.60 \\
\hline $\mathrm{P} 4$ & 0.92 & 0.25 & 0.12 & 0.92 & 0.25 & 0.46 & 0.60 \\
\hline $\mathrm{O} 1$ & 0.46 & 0.75 & 0.46 & 0.46 & 0.35 & 0.60 & 0.92 \\
\hline $\mathrm{O} 2$ & 0.12 & 1.00 & 0.21 & 1.00 & 0.58 & 0.33 & 0.40 \\
\hline F7 & 0.12 & 0.25 & 0.60 & 0.46 & 0.46 & 0.75 & 0.60 \\
\hline F8 & 0.12 & 0.25 & 0.60 & 0.46 & 0.46 & 0.75 & 0.60 \\
\hline $\mathrm{T} 7$ & 0.75 & 0.75 & 0.92 & 0.25 & 0.75 & 0.75 & 0.75 \\
\hline $\mathrm{T} 8$ & 0.92 & 0.05 & 0.60 & 0.35 & 0.17 & 0.75 & 0.60 \\
\hline P7 & 0.60 & 0.92 & 0.46 & 0.35 & 0.60 & 0.92 & 0.92 \\
\hline P8 & 0.75 & 0.75 & 0.35 & 0.92 & 0.03 & 0.92 & 0.60 \\
\hline $\mathrm{Fz}$ & 0.60 & 0.60 & 0.75 & 0.92 & 0.92 & 0.25 & 0.60 \\
\hline $\mathrm{Cz}$ & 0.35 & 0.75 & 0.12 & 0.75 & 0.46 & 0.75 & 0.92 \\
\hline $\mathrm{Pz}$ & 0.75 & 0.35 & 0.17 & 0.60 & 0.35 & 0.60 & 0.60 \\
\hline FC1 & 0.75 & 0.92 & 0.60 & 0.75 & 0.60 & 0.25 & 0.60 \\
\hline $\mathrm{FC} 2$ & 0.92 & 0.75 & 0.75 & 0.75 & 0.17 & 0.25 & 0.92 \\
\hline CP1 & 0.60 & 0.60 & 0.35 & 0.46 & 0.46 & 0.60 & 0.35 \\
\hline $\mathrm{CP} 2$ & 0.75 & 0.75 & 0.25 & 0.92 & 0.92 & 0.60 & 0.75 \\
\hline FC5 & 0.92 & 0.75 & 0.75 & 0.25 & 0.25 & 0.60 & 0.35 \\
\hline FC6 & 0.25 & 0.25 & 0.75 & 0.92 & 0.92 & 0.05 & 0.60 \\
\hline CP5 & 0.60 & 0.12 & 0.35 & 0.46 & 0.75 & 0.92 & 0.46 \\
\hline CP6 & 0.60 & 0.12 & 0.35 & 0.46 & 0.75 & 0.92 & 0.46 \\
\hline TP9 & 0.75 & 0.75 & 0.92 & 0.17 & 0.92 & 0.75 & 0.92 \\
\hline TP10 & 0.75 & 0.46 & 0.75 & 0.60 & 0.75 & 0.60 & 0.46 \\
\hline
\end{tabular}


Table 13. Entrainment at $\mathbf{1 5} \mathbf{~ m i n}$ (Placebo audio). Kruskal-Wallis $p$-significance.

\begin{tabular}{|c|c|c|c|c|c|c|c|}
\hline \multirow{2}{*}{ Electrode } & \multicolumn{7}{|c|}{ Bands } \\
\hline & Delta & Theta & Alpha & Beta & Gamma & Narrow $4 \mathrm{~Hz}$ & Narrow $14 \mathrm{~Hz}$ \\
\hline Fp1 & 0.92 & 0.35 & 0.60 & 0.17 & 0.92 & 0.12 & 0.35 \\
\hline Fp2 & 0.60 & 0.35 & 0.75 & 0.35 & 0.60 & 0.07 & 0.12 \\
\hline F3 & 0.35 & 0.25 & 0.46 & 0.03 & 0.35 & 0.60 & 0.03 \\
\hline F4 & 0.75 & 0.25 & 0.75 & 0.17 & 0.25 & 0.46 & 0.03 \\
\hline C3 & 0.46 & 0.12 & 0.25 & 0.35 & 0.17 & 0.25 & 0.35 \\
\hline $\mathrm{C} 4$ & 0.17 & 0.07 & 0.92 & 0.17 & 0.75 & 0.75 & 0.05 \\
\hline P3 & 0.92 & 0.12 & 0.05 & 0.07 & 0.17 & 0.25 & 0.46 \\
\hline $\mathrm{P} 4$ & 0.35 & 0.07 & 0.05 & 0.07 & 0.25 & 0.46 & 0.03 \\
\hline O1 & 0.46 & 0.17 & 0.07 & 0.07 & 0.17 & 0.17 & 0.46 \\
\hline $\mathrm{O} 2$ & 0.12 & 0.21 & 0.04 & 0.02 & 0.01 & 0.67 & 0.48 \\
\hline F7 & 0.35 & 0.35 & 0.35 & 0.17 & 0.75 & 0.75 & 0.25 \\
\hline F8 & 0.35 & 0.35 & 0.35 & 0.17 & 0.75 & 0.75 & 0.25 \\
\hline $\mathrm{T} 7$ & 0.60 & 0.25 & 0.46 & 0.60 & 0.92 & 0.75 & 0.25 \\
\hline $\mathrm{T} 8$ & 0.75 & 0.07 & 0.60 & 0.92 & 0.25 & 0.75 & 0.75 \\
\hline P7 & 0.92 & 0.17 & 0.07 & 0.17 & 0.12 & 0.12 & 0.05 \\
\hline P8 & 0.35 & 0.17 & 0.05 & 0.07 & 0.12 & 0.92 & 0.03 \\
\hline $\mathrm{Fz}$ & 0.46 & 0.25 & 0.92 & 0.12 & 0.92 & 0.75 & 0.07 \\
\hline $\mathrm{Cz}$ & 0.75 & 0.35 & 0.46 & 0.07 & 0.92 & 0.25 & 0.03 \\
\hline $\mathrm{Pz}$ & 0.25 & 0.07 & 0.05 & 0.07 & 0.12 & 0.12 & 0.35 \\
\hline $\mathrm{FC} 1$ & 0.75 & 0.35 & 0.35 & 0.92 & 0.92 & 0.46 & 0.60 \\
\hline $\mathrm{FC} 2$ & 0.92 & 0.35 & 0.92 & 0.75 & 0.35 & 0.92 & 0.12 \\
\hline CP1 & 0.92 & 0.17 & 0.03 & 0.12 & 0.12 & 0.17 & 0.05 \\
\hline $\mathrm{CP} 2$ & 0.92 & 0.25 & 0.05 & 0.25 & 0.92 & 0.12 & 0.25 \\
\hline FC5 & 0.35 & 0.17 & 0.35 & 0.12 & 0.12 & 0.46 & 0.25 \\
\hline FC6 & 0.35 & 0.25 & 0.92 & 0.35 & 0.35 & 0.92 & 0.05 \\
\hline CP5 & 0.46 & 0.05 & 0.07 & 0.60 & 0.46 & 0.12 & 0.35 \\
\hline CP6 & 0.46 & 0.05 & 0.07 & 0.60 & 0.46 & 0.12 & 0.35 \\
\hline TP9 & 0.35 & 0.17 & 0.07 & 0.46 & 0.12 & 0.35 & 0.05 \\
\hline TP10 & 0.25 & 0.17 & 0.35 & 0.17 & 0.75 & 0.60 & 0.05 \\
\hline
\end{tabular}

Table 14. Entrainment at $\mathbf{2 0}$ min / final (Placebo audio). Kruskal-Wallis p-significance.

\begin{tabular}{|c|c|c|c|c|c|c|c|}
\hline \multirow{2}{*}{ Electrode } & \multicolumn{7}{|c|}{ Bands } \\
\hline & Delta & Theta & Alpha & Beta & Gamma & Narrow $4 \mathrm{~Hz}$ & Narrow $14 \mathrm{~Hz}$ \\
\hline Fp1 & 0.12 & 0.07 & 0.75 & 0.03 & 0.25 & 0.35 & 0.25 \\
\hline Fp2 & 0.35 & 0.25 & 0.92 & 0.25 & 0.25 & 0.07 & 0.35 \\
\hline F3 & 0.46 & 0.35 & 0.92 & 0.03 & 0.75 & 0.92 & 0.07 \\
\hline $\mathrm{F} 4$ & 0.75 & 0.60 & 0.35 & 0.17 & 0.35 & 0.92 & 0.25 \\
\hline C3 & 0.92 & 0.12 & 0.75 & 0.92 & 0.75 & 0.75 & 0.75 \\
\hline $\mathrm{C} 4$ & 0.46 & 0.17 & 0.46 & 0.60 & 0.92 & 0.92 & 0.60 \\
\hline P3 & 0.60 & 0.25 & 0.35 & 0.46 & 0.12 & 0.92 & 0.46 \\
\hline P4 & 0.35 & 0.25 & 0.17 & 0.35 & 0.17 & 0.75 & 0.46 \\
\hline $\mathrm{O} 1$ & 0.92 & 0.60 & 0.17 & 0.46 & 0.12 & 0.75 & 0.35 \\
\hline $\mathrm{O} 2$ & 0.78 & 0.48 & 0.09 & 0.67 & 0.03 & 1.00 & 0.67 \\
\hline F7 & 0.92 & 0.12 & 0.46 & 0.07 & 0.92 & 0.60 & 0.35 \\
\hline F8 & 0.92 & 0.12 & 0.46 & 0.07 & 0.92 & 0.60 & 0.35 \\
\hline $\mathrm{T} 7$ & 0.92 & 0.60 & 0.46 & 0.60 & 0.25 & 0.92 & 0.46 \\
\hline $\mathrm{T} 8$ & 0.35 & 0.35 & 0.35 & 0.25 & 0.25 & 0.92 & 0.25 \\
\hline $\mathrm{P} 7$ & 0.35 & 0.35 & 0.25 & 0.46 & 0.17 & 0.92 & 0.46 \\
\hline P8 & 0.35 & 0.46 & 0.17 & 0.60 & 0.12 & 0.75 & 0.92 \\
\hline $\mathrm{Fz}$ & 0.46 & 0.46 & 0.92 & 0.17 & 0.35 & 0.92 & 0.46 \\
\hline $\mathrm{Cz}$ & 0.46 & 0.35 & 0.12 & 0.07 & 0.60 & 0.46 & 0.03 \\
\hline $\mathrm{Pz}$ & 0.35 & 0.46 & 0.25 & 0.35 & 0.25 & 0.75 & 0.75 \\
\hline FC1 & 0.60 & 0.35 & 0.35 & 0.75 & 0.92 & 0.92 & 0.12 \\
\hline $\mathrm{FC} 2$ & 0.75 & 0.60 & 0.75 & 0.75 & 0.25 & 0.92 & 0.60 \\
\hline CP1 & 0.46 & 0.35 & 0.25 & 0.17 & 0.05 & 0.60 & 0.92 \\
\hline CP2 & 0.46 & 0.35 & 0.25 & 0.25 & 0.12 & 0.75 & 0.75 \\
\hline FC5 & 0.46 & 0.46 & 0.75 & 0.46 & 0.25 & 0.75 & 0.46 \\
\hline FC6 & 0.92 & 0.25 & 0.92 & 0.60 & 0.92 & 0.92 & 0.60 \\
\hline CP5 & 0.92 & 0.12 & 0.35 & 0.75 & 0.75 & 0.75 & 0.92 \\
\hline CP6 & 0.92 & 0.12 & 0.35 & 0.75 & 0.75 & 0.75 & 0.92 \\
\hline TP9 & 0.92 & 0.75 & 0.25 & 0.75 & 0.75 & 0.60 & 0.46 \\
\hline TP10 & 0.75 & 0.25 & 0.25 & 0.35 & 0.60 & 0.92 & 0.75 \\
\hline
\end{tabular}




\section{Discussion}

In this paper, effects of commercially available BBs and a self-made stimulation were examined. We could not found any significant difference in the cognitive tests. This may be due to several factors, including wrong types of cognitive task were used or short duration of stimulation. It is true that the tests used in this study did not cover the whole spectrum of cognitive tasks (for instance planning and problem solving were not analyzed), but the commercial stimulation is advertised as "perfect for any mental task requiring focus and concentration", and the tests did require those capabilities.

We could not find either any significant difference in brain activity by means of EEG recording. It is possible that the size of the sample (6 per stimulation) was too small to examine group differences and/or the stimulation time was to short. The last possibility is the embedded noise with the stimulation. KASPRZAK (2011) reported significant changes using only BBs with no background sound. There were no side effects or adverse events noted by participants.

These results provide no evidence for improvements in cognitive function or changes in brain activity following binaural beat listening in a small sample of healthy adults after $20 \mathrm{~min}$. It is important to consider the possibility that one session is insufficient to produce a measurable effect, and further studies including several sessions of listening should be considered.

Because BBAS is a safe, non invasive, and potentially useful modality to entrain brainwaves (KASPRZAK, 2011) and to improve attention (KENNEL et al., 2010), this modality should be investigated farther using a larger sample.

Since subjects response can depend on baseline condition (ROSENFELD et al., 1997), population characteristic including mental health, psychological profile, QEEG, age, gender, and other baseline variables should be specific clearly. Measurement of QEEG and relevant hormones before and after stimulation would help clinical outcomes and improve our understanding of the mechanism. Hormones such as glucocorticoids and melatonin fluctuate during the day and affect arousal and thus EEG.

Finally, future studies should follow participants for an extended period of time to determine the effectiveness of this therapy over the time.

In further experiments it is necessary also use simple stimulation with only one layer of BBs trying to observe the fast following response. The EEG analysis for this signal is a difficult selection; future studies will have to calculate trends in order to know what happens along time. Also it is important to evaluate new parameters like hemispheric lateralization and evoked potentials.

\section{Acknowledgments}

This research was undertaken with the support of Centro de Acústica Aplicada y Evaluación No Destructiva (CAEND), Biomedical Technology Center (UPM) and Leonardo Torres Quevedo (CSIC Building).

We are grateful to Portuguese Society of Acoustics who organized VIII Iberoamerican Congress of Acoustics.

This paper was submitted at the VIII IberoAmerican Acoustic Congress held on October 32012 in Évora (Portugal).

\section{References}

1. Aftanas L.I., Golocheikine S.A. (2002), Non-linear dynamic complexity of the human EEG during meditation, Neuroscience letters, 330, 2, 143-6.

2. BARUŠs I. (2001), The Art of Science: Science of the Future in Light of, Journal of Scientific Exploration, 15, 1, 57-68.

3. Foster D.S. (1990), EEG and Subjective Correlates of Alpha-Frequency Binaural-Beat Stimulation Combined with Alpha Biofeedback, 1-34.

4. Kasprzak C. (2011), Influence of Binaural Beats on EEG Signal, Acta Fhysica Polonica, 119, 986-990.

5. Kennel S., Taylor A.G., Lyon D., Bourguignon C. (2010), Pilot Feasibility Study of Binaural Auditory Beats for Reducing Symptoms of Inattention in Children and Adolescents with AttentionDeficit/Hyperactivity Disorder, Journal of pediatric nursing, 25, 1, 3-11.

6. Lane J.D., Kasian S.J., Owens J.E., Marsh G.R. (1998), Binaural Auditory Beats Affect Vigilance Performance and Mood, Physiology \& Behavior, 63, 2, 249-252.

7. Lavallee C.F., Koren S.A., Persinger M.A. (2011), A Quantitative Electroencephalographic Study of Meditation and Binaural Beat Entrainment, The Journal of Alternative and Complementary Medicine, 17, 4, 351-355.

8. Oster G. (1973), Auditory beats in the brain, Scientific American, 229, 4, 94-102.

9. Ozimek E., Konieczny J., Sone T. (2008), Binaural perception of the modulation depth of AM signals, Hearing Research, 235, 1-2, 125-133.

10. Padmanabhan R., Hildreth A.J., Laws D. (2005), A prospective, randomised, controlled study examining binaural beat audio and pre-operative anxiety in patients undergoing general anaesthesia for day case surgery, Anaesthesia, 60, 9, 874-877.

11. Pérez M., Lago A. (2000), Escalas Magallanes de Atención Visual, Escalas Magallanes de Atención Visual, 30092. 
12. Reparaz C., Peralta F., Narbona J. (1996), El test de percepcion de diferencias (caras) como instrumento de medida de la atencion sostenida, Revista de ciencias de la educación, 166, 265-280.

13. Rosenfeld J.P., Reinhart A.M., Srivastava S. (1997), The effects of alpha (10-Hz) and beta (22-Hz) "entrainment" stimulation on the alpha and beta EEG bands: individual differences are critical to prediction of effects, Applied Psychophysiology and Biofeedback, 22, 1, 3-20.

14. Schwarz D.W.F., TAYlOR P. (2005), Human auditory steady state responses to binaural and monaural beats, Clinical Neurophysiology, 116, 3, 658-668.
15. Sedó M.A. (2004), Test de las cinco cifras: una alternativa multilingüe y no lectora al test de Stroop, Revista Española de Neurologia, 38, 9, 824-828.

16. Smith J.C., Marsh J.T., Brown W.S. (1975), Farfield recorded frequency-following responses evidence for the locus of brainstem sources, Electroencephalogr. Clin. Neurophysiol., 39, 5, 465-472.

17. Wahbeh H., Calabrese C., Zwickey H. (2007), Binaural Beat Technology in Humans: A Pilot Study To Assess Psychologic and Physiologic Effects, The Journal of Alternative and Complementary Medicine, 13, $1,25-32$. 